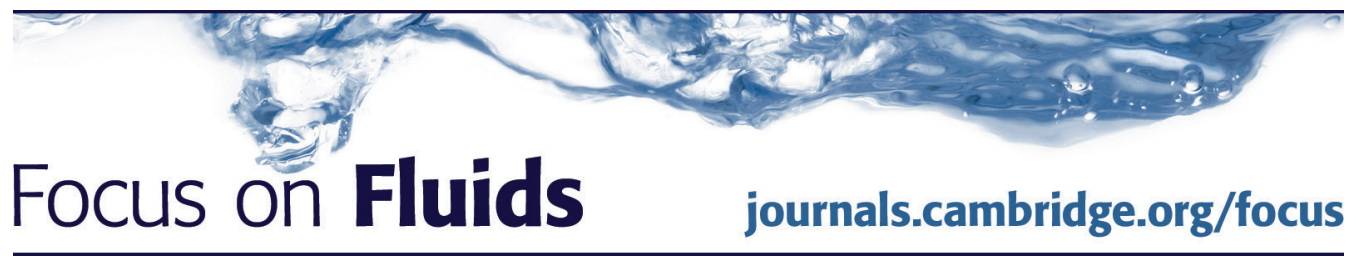

\title{
Dissipative distinctions
}

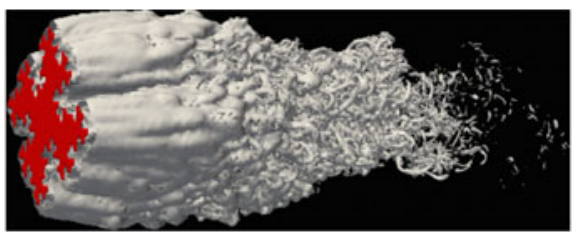

\author{
Ian P. Castro†
}

Aeronautics, Faculty of Engineering and the Environment, University of Southampton,

Southampton SO17 1BJ, UK

\begin{abstract}
There have been numerous studies concerning the possibility of self-similar scaling laws in fully developed turbulent shear flows, driven over the past half-century or so by the early seminal work of Townsend (1956, The Structure of Turbulent Shear Flow. Cambridge University Press). His and nearly all subsequent analyses depend crucially on a hypothesis about the nature of the dissipation, $\epsilon$, of turbulence kinetic energy, $k$. It has usually been assumed (sometimes implicitly) that this is governed by the famous Kolmogorov relation $\epsilon=C_{\epsilon} k^{3 / 2} / L$, where $L$ is a length scale of the energy-containing eddies and $C_{\epsilon}$ is a constant. The paper by Dairay et al. (J. Fluid Mech. vol. 781, 2015, pp. 166-195) demonstrates, however, that, in the specific context of an axisymmetric wake, there can be regions where $\epsilon$ has a different behaviour, characterised by a $C_{\epsilon}$ that is not constant but depends on a varying local Reynolds number (despite the existence of a $-5 / 3$ region in the spectra). This leads to fundamentally different scaling laws for the wake.
\end{abstract}

Key words: turbulence theory, wakes

\section{Introduction}

Townsend's classical self-preserving approach to characterising the behaviour of turbulent shear flows rests on the hypothesis that, at sufficiently large Reynolds numbers, the flow will eventually (i.e. sufficiently far downstream) 'forget' anything about how it was created and thus have a universal form determined solely by the necessary integral constraints. Analysis then leads to the well-known scaling laws for the mean velocity and turbulence stress fields, whose details depend only on the type of flow. For an axisymmetric wake, the analysis leads to a wake width governed by $\delta \sim\left(x-x_{o}\right)^{\lambda}$ and a decay in centreline velocity deficit governed by $u_{o} \sim\left(x-x_{o}\right)^{-2 \lambda}$ (with $\lambda=1 / 3$ ), where $x$ is the distance downstream from the wake-generating object and $x_{o}$ is a virtual origin. The turbulence statistics follow corresponding self-similar behaviour. Over the 60 years or so since Townsend's early work, there have been many, largely experimental, studies of all the possible flow types, which have assessed the adequacy of the classical scaling laws. Such studies

$\dagger$ Email address for correspondence: i.castro@soton.ac.uk 
are never very straightforward, and this is particularly true for the axisymmetric wake. To be confident about both $\lambda$ and $x_{o}$, it is necessary that the Reynolds numbers (both initial - set by the body geometry and upstream velocity - and local - set by $\sqrt{k}$ and $\delta$, say) are large enough to ensure that Townsend's 'memory' hypothesis and the Kolmogorov dissipation hypothesis are reasonable. In addition, there should be a sufficiently large range in $x$ downstream of the initial development region (a priori of unknown length). It is not easy to satisfy both requirements simultaneously.

There has thus been some controversy over whether the Townsend scalings hold. In fact, starting with the work of Bevilaqua \& Lykoudis (1978) over 35 years ago, there is mounting evidence that the geometry of the wake-generating body has a marked influence on the far-wake growth rate and turbulence even in regions where self-similarity is present, which leads to questions about whether the initial conditions really are ever forgotten. Much later, a similar result was found for plane wakes by Zhou \& Antonia (1995). Johansson, George \& Gourlay (2003) undertook a more complete analysis and showed that, for small Reynolds number, an additional scaling was possible (for which $\lambda=1 / 2$ ) and that both solutions can indeed be dependent on initial conditions. A numerical study was first done for a high-Reynolds-number case by Gourlay et al. (2001). Both they and Redford, Castro \& Coleman (2012) studied the spatially homogeneous but time-developing equivalent of axisymmetric wakes using direct numerical simulation (DNS). The latter showed apparently unequivocally that, for late enough times (corresponding to very far downstream in the spatially developing case), the classical $\lambda=1 / 3$ universal behaviour occurs, in which the multiplying constants (e.g. in the growth-rate relation for $\delta$ ) are truly independent of the initial conditions and $C_{\epsilon}$ is essentially constant.

It is especially crucial to recognise that all the extant work has assumed the adequacy of Kolmogorov's hypothesis (that the small-scale motions evolve much more rapidly than the time scale of the evolution of the whole flow), which led, with additional assumptions, to the famous equilibrium dissipation law, $\epsilon=C_{\epsilon} k^{3 / 2} / L$ (with $C_{\epsilon}=$ const.). The major objective of Dairay, Obligado \& Vassilicos (2015) is to 'establish the existence of a new non-equilibrium dissipation law', which assumes that, given a global Reynolds number set by the initial conditions, $C_{\epsilon} \sim R e_{l}^{-n}$, where $R e_{l}$ is a local Reynolds number. They do this for an axisymmetric wake, comparing their data with the scaling-law exponents that arise on the basis of this new dissipation law.

\section{Overview}

After a brief introduction to the arguments leading to the classical axisymmetric wake scalings in which $\lambda=1 / 3$, not least their reliance on the assumption that $C_{\epsilon}=$ const., Dairay et al. (2015) introduce their alternative non-equilibrium dissipation law (discussed more fully by Vassilicos (2015)), which states that $C_{\epsilon} \sim R e_{G}^{m} / R e_{l}^{n}$, where $R e_{G}$ is a global Reynolds number set by the initial conditions and $R e_{l}$ is a local one, which for an axisymmetric wake (as for grid turbulence) falls with distance downstream. From the equations of motion and on the basis of the similarity arguments by George (1989) (and see also Johansson et al. (2003)), this leads to the wake scaling laws derived by Nedić, Vassilicos \& Ganapathisubramani (2013), which can essentially be expressed as $\delta \sim\left(x-x_{o}\right)^{1 /(3-n)}$ and $u_{o} \sim\left(x-x_{o}\right)^{-2 /(3-n)}$, with $n=m=1$ (cf. $n=m=0$ for $\lambda=1 / 3$ ). Note that this value of $n$ and $m$ is the same as for a laminar wake $(\lambda=1 / 2)$, although it arises for different reasons. However, it can also arise (and does) when the classical Kolmogorov law is assumed for a fully turbulent, high-Reynolds-number wake characterised by large ratios of turbulence to 
viscous stress, provided only that the eddy viscosity is constant (Redford et al. 2012). The presence of $n=1$ scaling is thus not necessarily associated with low Reynolds number.

Dairay et al. (2015) examine their scalings by exploring the wake of an irregular (fractal-type) bluff plate with sharp edges, using both a wind tunnel experiment and a matching DNS. This is unusual, not only in that both approaches are used in the same work but also because the DNS is of a spatially developing wake (as in the experiment), rather than a time-developing one, with the generating body included in the computational domain. Whilst this allows capture of the near wake it has the inevitable consequence that the available downstream extent of the wake is somewhat limited. It is also unusual because of the use of a fractal-type wake-generating plate, which leads, in the very near field, to a ('multi-scale') flow having a mixture of wake-like and jet-like character. For the DNS, great care is taken to ensure that there is sufficient domain size, grid resolution and statistical convergence. For the experiments, the plate is placed in a low-turbulence wind tunnel at $\operatorname{Re}_{G}=U_{\infty} L_{b} / v=40000$, where $L_{b}=\sqrt{A}$, with $A$ the plate area. The measurements are made using hot-wire anemometry and extend to $x \approx 50 L_{b}$, whereas the DNS has $R e_{G}=5000$ and reaches approximately $x=100 L_{b}$.

The results suggest that, over most of the extent of the wind tunnel wake $(15 \leqslant$ $\left.x / L_{b} \leqslant 50\right), C_{\epsilon} R e_{l}^{n}$ with $n=1$ is more closely constant than is $C_{\epsilon}$. Actually, they show that a better fit requires $n \approx 0.77$. Further downstream $\left(55 \leqslant x / L_{b} \leqslant 100\right)$ the DNS data suggest a change to $n \approx 0.5$. To derive theoretical scalings for wake width and velocity deficit that have $n \neq 1$, the authors make a 'constant anisotropy' assumption - that the Reynolds shear stress and the turbulent kinetic energy profiles scale in the same way (but not with $u_{o}^{2}$ ). This is essentially a revised Townsend-George theory (Townsend 1976; George 1989), but includes the new 'non-equilibrium dissipation' law. (Note that only the latter is necessary for $n=1$.) The variations of $u_{o}$ and $\delta$ along the wake are shown to conform quite well to the new scalings, $u_{o} \sim\left(x-x_{o}\right)^{\alpha}$ and $\delta \sim\left(x-x_{o}\right)^{\beta}$, with $\alpha=-2 \beta=-2(1+n) /(3+n)$, albeit with different $n(<1)$ for the upstream and downstream halves of the $x$ region studied. Given that the local Reynolds number $(\sqrt{k} \delta / v)$ only falls to around 230 by $x=100 L_{b}$ in the DNS, and is very much higher in the wind tunnel, it is arguably difficult to claim that it is too small to expect the classical scaling to hold.

In addition to the increasing body of evidence that the value of $C_{\epsilon}$ can depend on initial conditions, Dairay et al. (2015) (following Nedić et al. (2013), along with the same group's work on grid turbulence) thus go much further and question the universality of Reynolds-number independence of $C_{\epsilon}$. No physical explanation is offered for why the $C_{\epsilon} R e_{l}^{n}=$ const. dissipation law might apply, but it does provide revised scaling laws for axisymmetric wakes that fit the present data.

\section{Future}

Although there is considerable evidence for the adequacy of the classical dissipation relation, it is apparent that it may be too simplistic, at more than one level. (Actually, it has long been recognised that $C_{\epsilon}$ is unlikely to be universal (Taylor 1935).) It is already clear that, whilst at sufficiently large Reynolds number $C_{\epsilon}$ may become constant, its precise value can depend on initial conditions (e.g. Sreenivasan 1998; Antonia \& Pearson 2000). Dairay et al.'s data seem even more revealing, in that, using only one wake-generating body, they show that $C_{\epsilon}$ is not even constant, but rather varies with local Reynolds number. The Kolmogorov law $\epsilon=C_{\epsilon} k^{3 / 2} / L$, with $C_{\epsilon}=$ const., is often seen as 'one of the cornerstone assumptions of turbulence theory' but Lumley (1992) remarked that the 'mechanism that sets the level of dissipation in 
a turbulent flow, particularly in changing circumstances', is worthy of further study. If the results of the Dairay et al. (2015) study can be shown to be typical of other high-Reynolds-number turbulent shear flows 'in changing circumstances', or indeed in axisymmetric wakes at significantly higher $R e_{l}$ than they could reach, the premonition implied by Lumley's remark will prove to have been prophetic. The matter is certainly worthy of more extensive study, not least because the non-equilibrium dissipation law seems to break the link between the presence of $-5 / 3$ spectra and classical cascade arguments. This new law seems to hold over much of any wake region that is likely to exist in real applications, so, even without any physical explanation, it would seem to be important. One can expect further experiments exploring the issue, aimed not least at finding whether (and if so, why) there is a final transition to a more classical scaling at some greater distance downstream, as Dairay et al. (2015) suggest, even though $\operatorname{Re}_{l}$ must continue to fall.

Finally, note that little is known about how the very-near-wake flow transitions to the region explored by Dairay et al. (2015). The process must surely be very dependent on the geometry of the generating body. The near-wake usually contains interesting and complex dynamics (e.g. the recent work of Rigas et al. (2015) and references therein). There would seem to be much scope for exploration of the various transition regions. And there remains the need for a physical explanation for the new non-equilibrium dissipation relation.

\section{References}

Antonia, R. A. \& Pearson, B. R. 2000 Effect of initial conditions on the mean energy dissipation rate and the scaling exponent. Phys. Rev. E 62, 8086-8090.

Bevilaqua, P. M. \& LYKoudis, P. S. 1978 Turbulence memory in self-preserving wakes. J. Fluid Mech. 89, 589-606.

Dairay, T., Obligado, M. \& Vassilicos, J. C. 2015 Non-equilibrium scaling laws in axisymmetric turbulent wakes. J. Fluid Mech. 781, 166-195.

GeORGE, W. K. 1989 The self-preservation of turbulent flows and its relation to initial conditions. In Advances in Turbulence (ed. W. K. George \& R. Arndt), pp. 39-73. Hemisphere.

Gourlay, M. J., Arendt, S. C., Fritts, D. C. \& Werne, J. 2001 Numerical modelling of inititially turbulent wakes with net momentum. Phys. Fluids 13, 3782-3802.

Johansson, P. B. V., George, W. K. \& Gourlay, M. J. 2003 Equilibrium similarity, effects of initial conditions and local Reynolds number on the axisymmetric wake. Phys. Fluids 15, 603-617.

Lumley, J. L. 1992 Some comments on turbulence. Phys. Fluids A 4, 201-211.

Nedić, N., Vassilicos, J. C. \& Ganapathisubramani, B. 2013 Drag and near wake characteristics of flat plates normal to the flow with fractal edge geometries. Phys. Rev. Lett. 111, 144503.

Redford, J. A., Castro, I. P. \& Coleman, G. N. 2012 On the universality of turbulent axisymmetric wakes. J. Fluid Mech. 710, 419-452.

Rigas, G., Morgans, A. S., Brackston, P. D. \& Morrison, J. F. 2015 Diffusive dynamics and stochastic models of turbulent axisymmetric wakes. J. Fluid Mech. 778, R2.

SreenivasAn, K. R. 1998 An update on the energy dissipation rate in isotropic turbulence. Phys. Fluids 10, 528-529.

TAYlor, G. I. 1935 Statistical theory of turbulence. Proc. R. Soc. Lond. A 151, 421-444.

Townsend, A. A. 1956 The Structure of Turbulent Shear Flow. Cambridge University Press.

Townsend, A. A. 1976 The Structure of Turbulent Shear Flow, 2nd edn. Cambridge University Press.

VAssilicos, J. C. 2015 Dissipation in turbulent flows. Annu. Rev. Fluid Mech. 47, 95-114.

Zhou, Y. \& Antonia, R. A. 1995 Memory effects in a turbulent plane wake. Exp. Fluids 19, $112-120$. 